\title{
Scene Matching Method for Children's Psychological Distress Based on Deep Learning Algorithm
}

\author{
Junli Su \\ Department of Elementary Education, Jiaozuo Normal College, Jiaozuo, Henan 454000, China \\ Correspondence should be addressed to Junli Su; sujunli@jzsz.edu.cn
}

Received 25 November 2020; Revised 22 January 2021; Accepted 25 January 2021; Published 3 February 2021

Academic Editor: Wei Wang

Copyright (C) 2021 Junli Su. This is an open access article distributed under the Creative Commons Attribution License, which permits unrestricted use, distribution, and reproduction in any medium, provided the original work is properly cited.

\begin{abstract}
In the process of children's psychological development, various levels of psychological distress often occur, such as attention problems, emotional problems, adaptation problems, language problems, and motor coordination problems; these problems have seriously affected children's healthy growth. Scene matching in the treatment of psychological distress can prompt children to change from a third-person perspective to a first-person perspective and shorten the distance between scene contents and child's perceptual experience. As a part of machine learning, deep learning can perform mapping transformations in huge data, process huge data with the help of complex models, and extract multilayer features of scene information. Based on the summary and analysis of previous research works, this paper expounded the research status and significance of the scene matching method for children's psychological distress, elaborated the development background, current status, and future challenges of deep learning algorithm, introduced the methods and principles of depth spatiotemporal feature extraction algorithm and dynamic scene understanding algorithm, constructed a scene matching model for children's psychological distress based on deep learning algorithm, analyzed the scene feature extraction and matching function construction of children's psychological distress, proposed a scene matching method for children's psychological distress based on deep learning algorithm, performed scene feature matching and information processing of children's psychological distress, and finally conduced a simulation experiment and analyzed its results. The results show that the deep learning algorithm can have a deep and abstract mining on the characteristics of children's psychological distress scenes and obtain a large amount of more representative characteristic information through training on large-scale data, thereby improving the accuracy of classification and matching of children's psychological distress scenes. The study results of this paper provide a reference for further researches on the scene matching method for children's psychological distress based on deep learning algorithm.
\end{abstract}

\section{Introduction}

Children's psychological distress refers to the distress caused by the deviation of children's psychological development from normal in perception, thinking, emotion, behavior, personality, attention, habits, and development, which clinically often manifests as special developmental disorders, extensive developmental disorders, childhood hyperactivity, conduct disorders, emotional disorders, tic disorders, and behavior disorders [1]. Psychological distress has a great negative impact on children, which not only affects their normal study and life but also leads to emotional and behavioral problems and may make severe mental breakdown, or even suicide; in addition, if children suffer from repeated psychological distress and cannot cope with it, this will affect the normal development of their future personalities [2]. Therefore, children's psychological distress should arouse great attention from family, school, and society. A psychological scene is a subjectively constructed environment, and the information scene created by medium is as important as natural environment in which people express their behavior [3]. Scene matching in the treatment of psychological distress can prompt children to change from a third-person perspective to a first-person perspective and shorten the distance between scene contents and child's perceptual experience, so as to achieve the scene creation containing intelligent matching and element dissemination and have 
great significance to the treatment of children's psychological distress [4].

As a part of machine learning, deep learning can perform mapping transformations in huge data, process huge data with the help of complex models, and extract multilayer features of the data. Typical deep learning models mainly include deep belief networks, stacked autoencoders, and multilayer convolutional neural networks [5]. Deep learning is essentially a deep abstract mining of data features, and a large amount of more representative feature information will be obtained through large-scale data training, thereby improving the accuracy of classification or prediction [6]. In deep learning methods, the focus is on the problem, the feature vector is determined, and data processing is realized by training and extraction, and deep learning methods can help people carry out multilevel analysis of data representation [7]. Deep learning is essentially a process of continuous feature description, that is, a hierarchical feature extraction process. The more features that are extracted and more accurate, the easier it is to achieve overfitting; the fewer and more fuzzy the feature extraction, the easier it is to achieve under fitting. Therefore, how to use massive data to train model parameters so that the training results reach the peak accuracy is the difficulty of deep learning [8].

On the basis of summarizing and analyzing previous research works, this paper expounded the research status and significance of the scene matching method for children's psychological distress, elaborated the development background, current status, and future challenges of deep learning algorithm, introduced the methods and principles of depth spatiotemporal feature extraction algorithm and dynamic scene understanding algorithm, constructed a scene matching model for children's psychological distress based on deep learning algorithm, analyzed the scene feature extraction and matching function construction of children's psychological distress, proposed a scene matching method for children's psychological distress based on deep learning algorithm, performed scene feature matching and information processing of children's psychological distress, and finally conduced a simulation experiment and analyzed its results. The detailed chapters are arranged as follows: Section 2 introduces the methods and principles of depth spatiotemporal feature extraction algorithm and dynamic scene understanding algorithm; Section 3 constructs a scene matching model for children's psychological distress based on deep learning algorithm; Section 4 proposes a scene matching method for children's psychological distress based on deep learning algorithm; Section 5 conduces a simulation experiment and analyzes its results; Section 6 is conclusion.

\section{Methods and Principles}

2.1. Deep Spatiotemporal Feature Extraction Algorithm. Compared with shallow learning, deep learning has two or more hidden layers; in order to discover the distribution characteristics and potential attributes of the data, it converts the combination of low-level features into high-level abstract features. The core is to make the data from the concrete through nonlinear transformations; from abstract, low-level to high-level, the mathematical relationship between them can be expressed as follows:

$$
f(x)=k\left[m_{x} a(x)-n_{x} b(x)\right],
$$

where $f(x)$ is hidden feature; $a(x)$ is nonlinear activation function; $b(x)$ is the common activation function; $k$ is the actual output ratio before and after hidden feature transformation; $m_{x}$ is weight matrix; $n_{x}$ is error vector.

$H_{i j}$ is used to minimum norm sparse optimization to obtain the sparse weight direction value, and the key element to optimize and update the extracted features is

$$
H_{i j}(x)=\gamma \sum_{i=1}^{n} \sum_{j=1}^{m} \frac{f(x)-s(i)}{f(x)-t(j)},
$$

where $\gamma$ is the number of factors; $s(i)$ is the size of node $i ; s(j)$ is the size of original node $j ; n$ and $m$ are the number of features and samples, respectively.

The downsampling layer is to perform local averaging and secondary extraction of the feature scene of upper layer. This operation can not only reduce the amount of data, but also retain useful information, reduce the calculation time, and reduce the sensitivity of the network output to displacement and deformation. Assuming the feature scene of $i$-th convolutional layer $P_{i}$, then

$$
P_{i}=\int_{i=1}^{n} \frac{u_{i} \cdot X_{i}}{v_{i} \cdot Y_{i}}
$$

where $X_{i}$ is activation function; $Y_{i}$ is the set of input feature maps of previous layer; $u_{i}$ is the weight of learnable convolution kernel; $v_{i}$ is the only additive bias of convolution feature scene.

The result of the pooling layer is input into the classification layer for classification, and the classification layer calculates the predicted probability distribution $W(y \mid x)$ of the label corresponding to the input sequence through logistic regression:

$$
W(y \mid x)=\alpha \ln e_{x}+\beta \ln e_{y},
$$

where $e_{x}$ and $e_{y}$ are the classification numbers of $x$ and $y ; \alpha$ and $\beta$ are the weight values when the model predicts different classification numbers.

Given a set of randomly selected training data, since the conditional probability value of the hidden unit only depends on the distribution of the explicit unit, the conditional probability of the hidden unit is

$$
E_{i}=\int_{i=1}^{n}\left[\rho \frac{S_{i}}{P_{i}}+\sigma \frac{T_{i}}{P_{i}}\right],
$$

where $E_{i}$ is the connection strength between visible unit and hidden unit; $S_{i}$ is the bias of visible unit $i$; $T_{i}$ is the bias of hidden unit $i ; \rho$ is the state of visible unit; $\sigma$ is the state of hidden unit.

2.2. Dynamic Scene Understanding Algorithm. Assuming the neural network parameters are as follows: $z_{i}(i=1,2, \ldots, n)$ is input sample, then $z_{i}^{e}$ is the component of each sample, $q_{i}^{e}$ is 
the weight of the $i$-th neuron, then its input and output will satisfy the following equation:

$$
\frac{\mathrm{d} z_{i}}{\mathrm{~d} t}=\sum_{i=1}^{n} \frac{z_{i}^{e}-g\left(z_{i}\right)}{q_{i}^{e}-l\left(z_{i}\right)}
$$

where $t$ is the weighted cumulative sum of the input; $g\left(z_{i}\right)$ is a function that slows down the speed of $z_{i} ; l\left(z_{i}\right)$ is a monotonically rising nonlinear function.

When the state of all neurons on the visible layer is given, the probability of a certain neuron on the hidden layer is activated; that is, the posterior probability is calculated:

$$
u_{i}=\delta r_{i} g\left(z_{i}\right)+\varphi c_{i} l\left(z_{i}\right),
$$

where $\delta$ is the bias of hidden layer; $r_{i}$ is the bias of the $i$-th neuron in hidden layer; $\varphi$ is the weight matrix between visible and hidden layer; $c_{i}$ is the link weight between the neuron and the $i$-th neuron in visual layer.

Assume that $U(i)$ is used to represent the category of sample $i$ and the probabilistic collaboration space is

$$
U_{i}=\frac{\sqrt[k]{u_{i} E_{i}-h F}}{d^{k}},
$$

where $d_{k}$ is the matrix composed of all the features; $E_{i}$ is the matrix composed of all the features; $h_{i}$ is the class label; $F_{i}$ is the linear space formed by the collaborative representation of all samples.

There are usually averaging methods, voting methods, and learning methods for combining multiple learners. The averaging method averages the outputs of multiple learners to obtain the final prediction. When the average calculation is a simple arithmetic average:

$$
S(x)=\frac{1}{n} \prod_{i=1}^{n} U_{i}\left[\frac{E_{i}}{d i}-W_{i}(x)\right],
$$

where $S(x)$ is the final prediction result, $n$ is the number of learners, $E_{i}$ is the prediction result of a single learner, and $W_{i}$ is the weight of a single learner.

Only using the normalization method can adjust the input distribution of each layer and speed up training, but it will change the input feature expression. Therefore, batch normalization adds two parameters to scale and translate the normalized value to maintain the model expressive ability:

$$
f^{(x)}=u_{i} h^{(x)}-z_{i} v^{(x)},
$$

where $f(x)$ is the $x$-th input in a batch of inputs, $h(x)$ is a tiny constant added to ensure numerical stability, and $v(x)$ is the mean matching degree of the batch of inputs.

\section{Scene Matching Modeling for Children's Psychological Distress Based on Deep Learning Algorithm}

3.1. Feature Extraction of Children's Psychological Distress Scenes. Psychological research shows that a healthy psychology is essential for children's growth and healthy personality, but many parents in real life do the opposite. The result is that children's psychological endurance is too low to a certain extent, so that they cannot withstand the pressure and setbacks in life; even a small matter can make them depressed, decadent, self-destructive, or afraid. Family size and family relationships are closely related to the development of children's psychological health. A set of concise and artistically beautiful scene matchings should be a good medium for coordinating the entire activity space, and a guiding system design with formal aesthetics should guide the viewers to produce correct behaviors while bringing them beautiful visual experience and enjoyment. Children will have success in the process of learning and living, and they will inevitably encounter failures; they will have positive emotions, but they cannot avoid negative emotions. Therefore, parents should actively create opportunities for children to match scenes, encourage children to go to communities and playgrounds to help them experience the joy of activities with others, and consciously guide children to follow the rules of communication and learn to pay attention to the emotions of others. They change, experience, and understand the emotions of others and let them experience the psychological state of others in the game interaction, so as to correct the child's aggressive behavior [9].

The scene matching of the guidance system must have an aesthetic function while transmitting information, which should become an important part of the entire public activity space. This kind of design art with practical functions can not only have a positive influence on children's behavior, but also have culture and spirit and can have a comprehensive and positive influence on children Figure 1. The perspective of children in the process of design is to make correct judgments on their own ability of a certain behavior which is not only beneficial to children's psychological health but also has a great impact on learning how the system can better met the needs of children. Research and design can only be a sign but should be a sign that fully integrates the surrounding environment and can correctly guide children's behavior. Therefore, the important significance of designing a scene matching system is to stimulate children's exploration spirit while ensuring safety and to create a good environment for exercising children's physical abilities. This requires designers to design systematic scene matching from the perspective of children in the process of design and at the same time considers more humanistic care and truly considers how the system can better meet the needs of children.

Children's memory of repeated scenes and cochanging target positions in real scenes is explicit. The results of the study using simple stimulus matching found that there was no difference between the correct recall rate and the random level of repeated matching and covariation target positions, indicating that the memory is implicit. The different results obtained by using real scenes and simple stimulus matching as research materials may be due to the fact that real scenes can be quickly classified and defined and provide expectations about spatial locations and constituent objects and then guide attention to the scene area related to the task [10]. Moreover, the semantic memory of the scene content such as the scene category and object placement rules in the real scene can also 


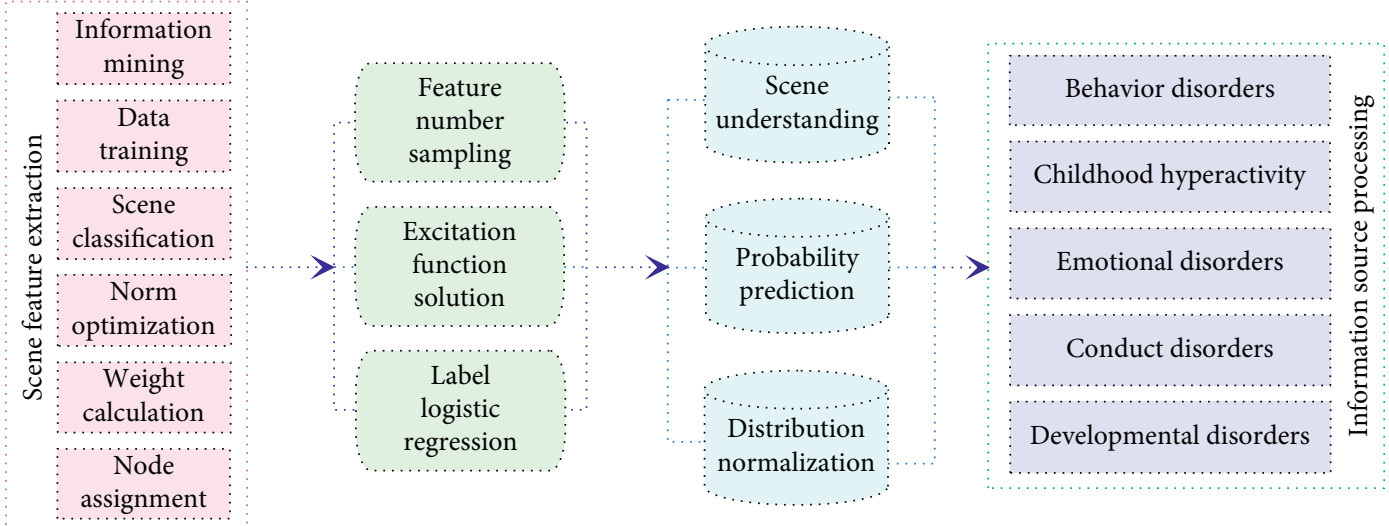

FIGURE 1: Scene matching framework for children's psychological distress based on deep learning algorithm.

promote the acquisition of clue benefits. Children can obtain background cue effects, and memory can guide their attention; therefore, in real scenes, explicit memory plays a role in the background cue effect. This shows that when human beings come to this world, they can arm themselves with skilled learning mechanisms and develop rules that exist in the objective world. Using real scenes as the search background is an essential extension of this work. It shows that children can sensitively obtain background structure information and the covariation relationship between background and target in a complex real environment, and this memory guides the distribution of attention and becomes more effective with experience.

\subsection{Matching Function Construction of Children's Psycho-} logical Distress Scene. From the school's perspective, teachers can change their educational concepts, pay attention to children's psychological changes and emotional reactions, attach importance to the guidance and assistance of psychological distress, deliberately understand children's psychological characteristics, and strive to improve teaching and education methods. Teachers can try to use psychological education methods to deal with children's problems, not just moral education methods; in education and teaching, teachers should always pay attention to the possible impact of their words and deeds on children and try their best to avoid harm to children's self-esteem. To maximize the elimination of teacher-induced psychological damage, schools can set up a children's activity room, a psychological consultation room, and a love mailbox, so that children with psychological distress can go to rest, and if they have something to tell, they can get help from a psychological teacher. In addition, schools can conduct courses with positive psychological guidance to form a targeted education method. The courses with positive psychological guidance must have some learning and interesting scenes; children can use these scenes, such as setting up situations, assigning roles, and discussing methods. They make these psychologically troubled children have more psychological tolerance, allow them to have an optimistic attitude, and get along with people around them and being friendly, control their emotions, and avoid being pessimistic when encountering certain things.

The level of group relationship in the peer relationship, that is, peer status, reflects the degree to which peers like or dislike individuals. Previous studies have shown that peer acceptance of children has a lower incidence of internal and external problems due to the harmonious relationship between peers and more support. The peers refused to experience more loneliness, poorer academic adaptation, more aggressive behaviors, and violations of discipline. Figure 2 shows the matching function construction of children's psychological distress scene. There are plants, such as trees and flowers, which are symbols of vitality, energy, and beauty, and daily necessities, such as food and lighting, which are expressions of achievement and hope. The lack of plants and daily necessities shows that the peers reject the child's lack of energy and psychological state. In addition, parents with a good parent-child relationship can teach their children how to deal with peer relationships, such as how to deal with disputes and avoid embarrassment, and encourage children to learn to tolerate others and guide children to cope with pressure from others [11]. Children rejected by their peers may not be able to get support from family members due to insufficient parent-child relationship. Their inner conflicts cannot be resolved by external forces and all these help children develop good peer relationship and they are trapped in their own world and have difficulty communicating with others.

In scene matching, when children complete a task alone or in a team, they face the physical difficulties they must face during their growth. This is good for the establishment of their independent personality, and a good scene matching will also help and the growth of his life has a certain promoting effect. Scene matching is originally done by a team and the emotions generated in this process are unmatched by other methods. At the same time, the emotions cultivated in the treatment of this adversity will be valued and cherished, and they will also get to know a group of people who are treated as good friends and partners. In the storyline, no matter how many people you know around you, you always have to contact strangers. This means that children are an attack directed at oneself and can better understand the 


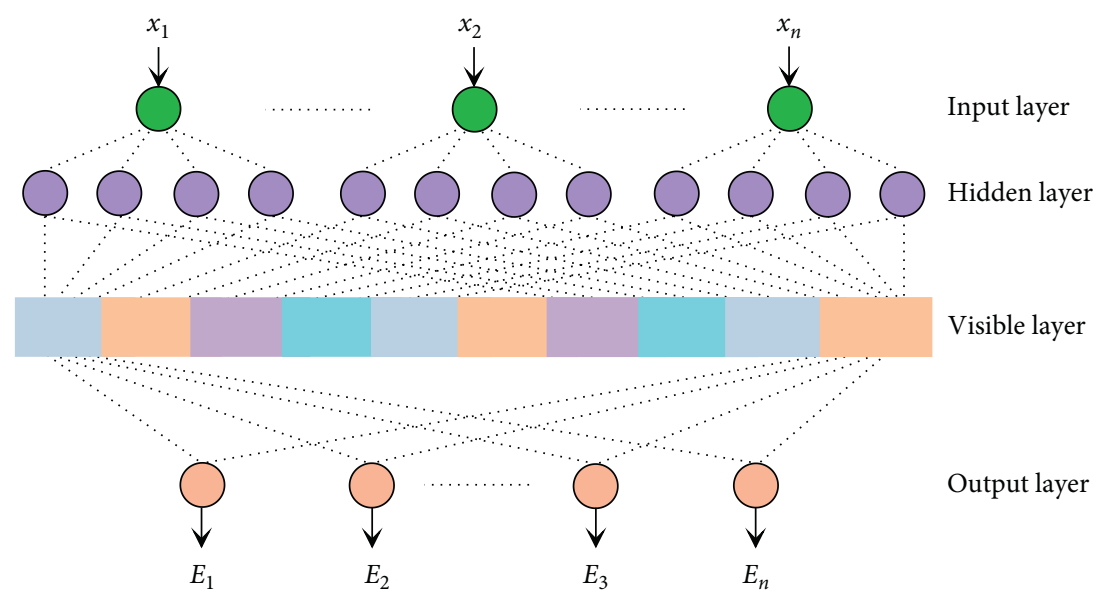

FIGURE 2: Matching functions construction of children's psychological distress scene.

psychological states of others' thoughts, which are designed to realize the semantic level question-answering system. Through this independent contact, the treated person will be able to face the unknown world independently and able to deal with the world and face it independently in the future. Therefore, the pain and insecurity that the treated person needs to bear the treatment process and the recovery period are still and issue the children themselves need to bear. Of course, the setting of the care link in the plot scene is to allow more people to care to reduce the loneliness, boredom, and helplessness of the treated person. Through the completion of a task, a partnership has been established and their care and blessings are extremely important for the recovery of the disease [12].

\section{Scene Matching Method for Children's Psychological Distress Based on Deep Learning Algorithm}

4.1. Information Source Processing of Children's Psychological Distress Scenes. Human cognitive ability mainly includes memory ability, attention, perception ability, imagination, and thinking ability. Predecessors have studied the obstacles of students in learning and divided them into academic obstacles and developmental obstacles. Developmental obstacles generally include insufficient memory, defects in perception, unusual thinking skills and language flaws, and flaws in the ability to judge numbers. Human cognition is a stage of rapid development in childhood, and children's cognitive abilities should be paid more attention to in order to promote the healthy and comprehensive development of children [13]. Only in this way can children be encouraged to learn actively and independently after entering primary school, and they want to master more from the heart. For adults, children should always be encouraged and affirmed in their daily study and life, actively praised for their correct behavior, and promoting the further development of their initiative. Children's confidence occupies an important position in personal self-awareness, which is a powerful kinetic energy that can mobilize people's enthusiasm and promote people's willingness to take the initiative.

The deep learning algorithm experimental platform, with cloud computing, and big data as the technical background, with artificial intelligence, machine learning, and deep learning algorithm as the core technology, realizes training in artificial intelligence fields such as speech recognition, image recognition, and natural speech processing. With the increase of the number of iterations, both the feature extraction and the average reconstruction error have a good improvement in the matching of children's psychological distress scenes. The degree of confidence network model can be carried out through unsupervised training in the pretraining stage and does not require a large amount of label data, which greatly improves the adaptability of the model Figure 3. In the pretraining process, the selection of parameters can speed up the convergence and reduce the computational complexity [14]. Unsupervised training improves the effectiveness of training parameters and prevents the calculation results from entering the local optimum instead of the global optimum. In this learning method, a large number of training samples constitute a sample set, and each training sample contains an input value and an expected output value. The realization of the supervised learning function is mainly to build a model through deep learning algorithm and then compare the prediction results generated by the model with the final data processing results and continuously optimize and adjust the model according to the deviation of the two to improve the model prediction results.

Defects in social emotional ability are the core of children's social psychological distress. In the field of normal children's social emotional ability training, the methods and courses are quite mature and suitable for children with psychological distress, but they are very dependent on the development of children's psychological theory and environmental hypotheses. The use of modern information media to other information technology media is conducive to more accuracy, and children can increase the influence of 


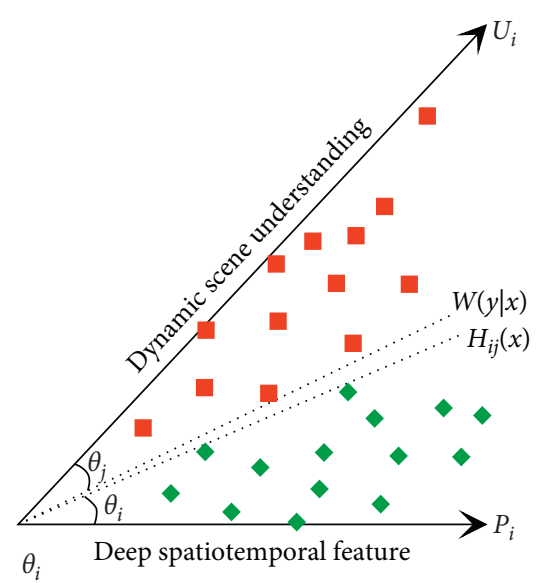

(a)

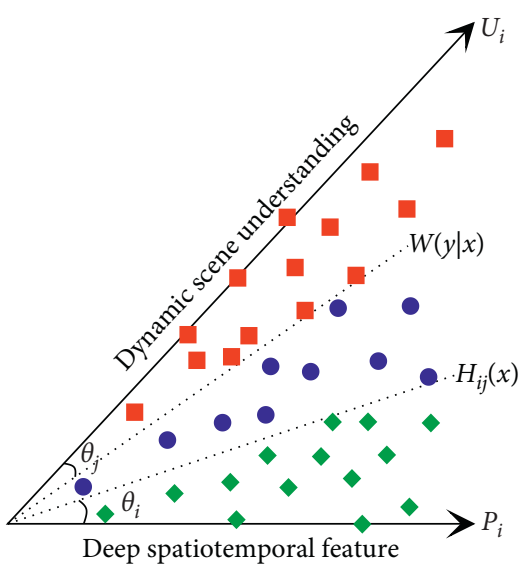

(b)

FIGURE 3: Normalized results of hidden and visible layer of deep learning algorithm. (a) Hidden layer. (b) Visible layer.

psychological education in life. Deep learning is a hierarchical feature extraction process, which is often used for feature extraction and classification and recognition as the input connection classifier. The intervention of children's psychosocial and emotional abilities requires the joint efforts of children, schools, and parents, which requires intervention from multiple perspectives such as the environment, curriculum, and intervention, and it is a continuous process. Compared with the actual environment of the intervention scene matching intervention, although it has the advantages of safety, economy, and high efficiency, many researchers are worried about its ecological validity. Practice has proved that the social emotional ability training courses have achieved great success in the field of normal children, but the interactive vehicles used in the commonly used intervention methods in these courses, such as pictures and story scripts, are very dependent on children's psychological theory, hypothetical environment, and imagination. In addition to that, children's psychological distress lacks interest in interacting with others and society, and their attention and cooperation abilities are also flawed. They often fail to cooperate with classmates and teachers to complete the course training, making the intervention fail to achieve the desired effect.

\subsection{Feature Matching of Children's Psychological Distress} Scenes. Many psychological distresses of today's children are caused by the influence of information technology media such as the Internet and digital television. In the online world and scene plots, children can find many partners with similar behaviors and ideas, forming a sense of belonging and dependence. However, due to the illusory nature of the Internet and digital scene and the unilateral nature of communication, children's society opportunities for communication are greatly reduced, so that they gradually break away from the true companionship. Therefore, the contradiction between the desire for communication and psychological lock-in hidden deep in the heart has been exacerbated. This is also a new problem faced by modern psychological education due to information technology. The use of modern information media to provide individualized psychological education to children can increase the influence of psychological education in life. It can also better detect potential serious psychological distress through information technology media and take appropriate educational countermeasures in time. For children with general psychological distress and severe psychological distress, further help and treatment are provided. At the same time, the use of other information technology media is conducive to more accurate and convenient establishment of school psychological education files and scene matching. It is especially effective for some students who are depressed and anxious due to improper learning methods, poor interpersonal communication, and poor psychological adjustment ability, which affect normal study and life [15].

In this method, the feature quality needs to be determined first, which directly determines the development efficiency of the final learning results, which is necessary to use semantically presented intersecting vectors to expand the research. This method can improve the dimensionality of the entire calculation process and avoid actual phenomenon of increased efficiency; this process can be realized by means of principal component analysis and deep learning algorithm Figure 4. Deep learning methods can perform local averaging and secondary extraction, and children can obtain the guidance and assistance of psychological distress. The lack of plants and daily necessities shows that the peers reject the child's lack of energy and psychological state. At present, deep learning algorithm has been widely used, including image recognition, speech recognition, and language processing. Because they are applied in different fields, their research focuses are also different and part of the focus is on system discovery and part of the focus is on nonlinear model establishment [16]. The clustering algorithm usually merges the input data according to the center point or layering the data and finds the internal structure of the data to classify the 


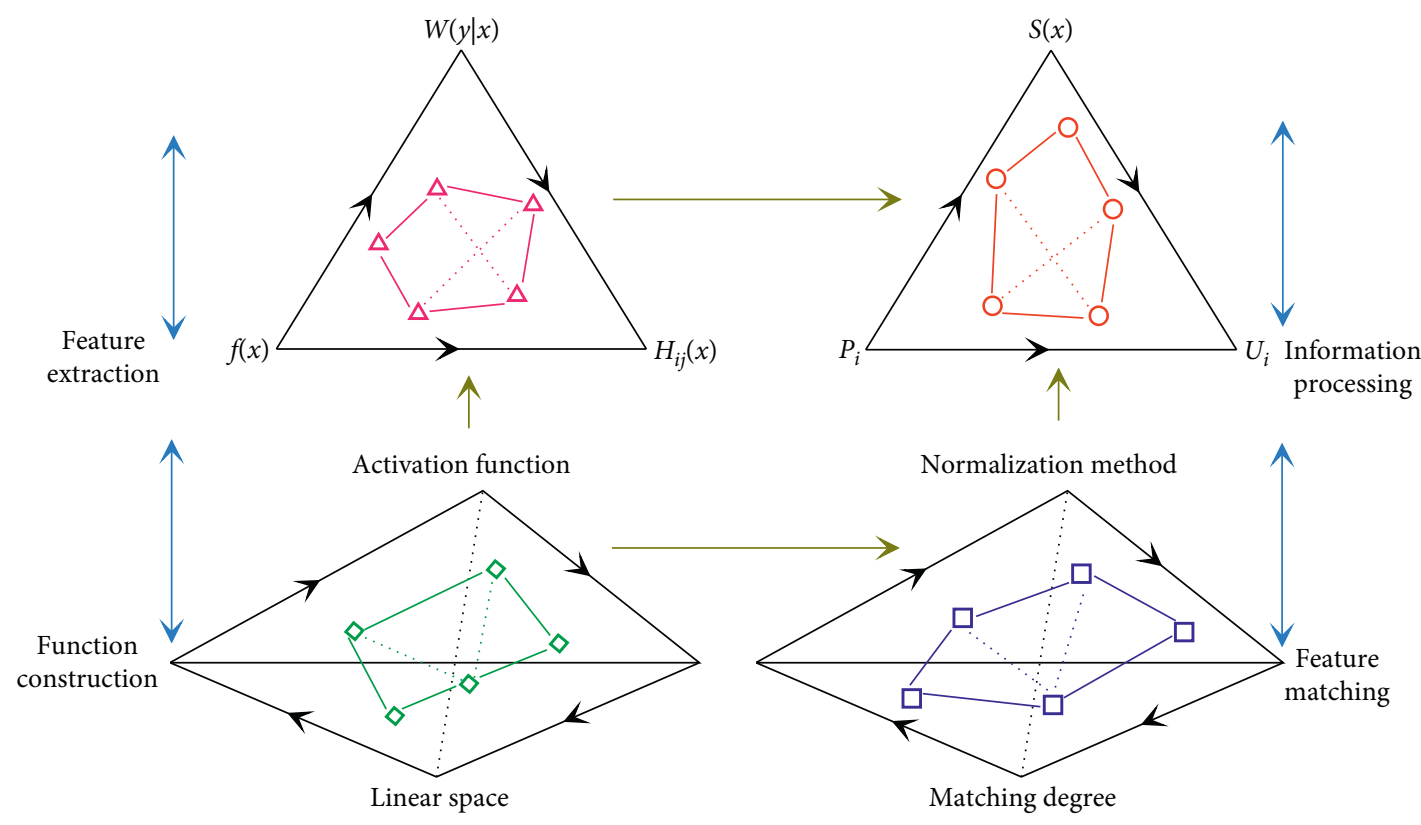

FIGURE 4: Scene matching flowchart for children's psychological distress based on deep learning algorithm.

data to the greatest extent. Association rule learning is a deep learning algorithm to improve the accuracy of decisionmaking. Through data analysis, the internal correlation between data is grasped, so as to determine the relationship between variables and grasp the rules behind the data. Similar to the clustering algorithm, the dimensionality reduction algorithm is also an attempt to obtain the internal structure of the data through the algorithm.

For children, school life is an important environment for their socialization and the impressions these children convey are rooted in the subtle influence of teachers' rules in school daily life. The good behavior of children in this situation is often the behavior that teachers expect them to perform; the role played by children is often the role that teachers expect them to play for teachers' cultivation of children's good behaviors. In essence, there is an operational agreement between the two, that is, the performer's satisfaction with the audience's expectations. Social life is complex and diverse, and this makes the cast members assume multiple roles. Therefore, there is a teacher-student relationship between the educated and the educator between children and their parents and there is also a parentchild relationship between parents and children. The role characteristics of children in the parent-child relationship have become an important factor affecting children's current behavior, which is also an important reason for the differences in their behavior. The teacher-student relationship is a derivative of social culture, which depends on the development and maintenance of educational activities in the teacher-student relationship. The role image of children is constantly being institutionalized into the image of the educated and in the parent-child relationship. The interaction between parents and children is not only educational activities but also parenting activities, games, and so on. In addition to being educated, children are also fostered and game participants.

\section{Simulation Experiment and Result Analysis}

5.1. Simulation Experiment Design. Among the factors that affect children's psychological distress, social cultural factors are the main factor, which includes the comprehensive effect of various factors such as urban and rural cultural differences, multimedia culture, and multiculturalism. Analysis of these factors can help solve children's psychological distress problems and promote their healthy growth and development. The coexistence of multicultural forms has established multiple value scales for all areas of social life, and there are even multiple value judgment standards for the same thing, and each value standard can find a reasonable basis from its own subordinate culture. In the noise of multiple values, it is difficult for adults to form a sense of belonging to a certain culture and fall into the confusion of value choices; this confusion naturally affects the surrounding children, and their value choices are more guided by adults. Growing up in such a cultural environment, how to cultivate children's cultural feelings and cultivate and explore their imagination and creativity are important issues that need to be resolved urgently [17]. There are many problems that need to be solved urgently, such as their psychological and behavioral problems, their studies, conduct, and interpersonal relationships. Through this independent contact, the use of modern information media to classify the data to the greatest extent and cultivate children's cultural feelings cultivates and explores their imagination. Regarding the education of migrant children, the rule-based method is to resolve conflicts by themselves in actual situations. Parents and teachers need to understand relevant knowledge, early attention and scene matching, to prevent these psychological problems from aggravating and becoming psychological disorders or even psychological illnesses. The matching training accuracies and test accuracies of four typical children's psychological distresses (solitary scene matching, 
social scene matching, entertainment scene matching, and learning scene matching) are shown in Figure 5.

The development of semantic analysis and recognition provides new directions for solving related problems. However, the current research on automatic question answering systems has not reached the level of semantic recognition. Therefore, to realize the semantic level question system, current common knowledge based on application fields studies the semantic representation of knowledge. An object-oriented semantic knowledge provided the basis for question-answering system and related research (Figure 6). The principle of knowledge base based on structured query is to transform the questions raised by natural language into structured query sentences and mainly use methods based on rules, keywords, synonyms, and semantic analysis to transform the question text. The perception stage is the processes in which all the feature maps are expanded, its adjacent layers were closely related, and the dependence on the environment cannot be satisfied. The rule-based method is to scene questions to predicate through artificially constructed rules, such as predicting related questions based on a set of question patterns. However, the recall rate of this type of method is low due to the limitation of the scale of manual construction rules and the knowledge base system is an entity that stores knowledge, which is a product of the combination of database technology and is the foundation of artificial intelligence application systems.

Children with a high level of theory of mind can better understand the psychological states of others' thoughts, intentions, and emotions, and in the process of interacting with other children, they will also adopt more acceptable strategies to better meet the needs of others. Children who use this strategy show that they have taken into account the interests of both parties and considered the acceptable degree of the solution by both parties, so, in a sense, this is a more scientific method. For conflicts in hypothetical situations, negotiation is the main choice, while in actual conflict situations children are more inclined to adopt coercive strategies. The second is that, for the third-party strategy, the parents' choice scores higher, but, in the two actual scenarios, they can only be classified into other strategies. This means that children are more willing to resolve conflicts by themselves in actual situations, rather than seeking help from teachers or parents as parents imagine. The above difference may be caused by the difference between the two measurement methods. Parents may choose strategies based on children's daily performance, ignoring the situational factors in the premise; children need to choose a strategy based on actual conflict situations and specific problems, and in these two story situations, may be everyone feels that there is no need for children to seek help from a third party, and they can resolve the conflict by themselves [18].

5.2. Result Analysis. The object relationship theory believes that, in the early development of the individual, the object relationship is underdeveloped, the empathy relationship and the empathy function are impaired, and the dependence on the environment cannot be satisfied, so anger and narcissistic attack occur. Attacks directed to the inside result in anxiety and depression, while attacks directed to the outside often result in aggressive behavior, violation of discipline, and antisocial behavior. This shows that the arms that are too long may only reflect the presence of unconsciously driven aggressiveness, but the direction of the attack cannot be determined, and it may be an attack directed at oneself, depression, or an attack directed outwards. For conflicts in hypothetical situations, negotiation is the main choice, while in actual conflict situations children are more inclined to adopt coercive strategies. Their overactive, disciplinary, and aggressive behaviors have not been corrected in time and turned into internalized manifestations Figure 7. As they grow older, internalized manifestations can be reversed when the transform is into explicit behavior [19]. The treatment goals for children with psychological distress include increasing self-control and behavior inhibition, reducing disruptive behaviors, developing effective communication behaviors, problem-solving skills and problemsolving skills, improving interpersonal relationships, reducing their peers' rejection of them, and increasing selfconfidence, reduce the troubles of parents and family, and increase the compliance with parents, the completion rate of task operations, and the accuracy rate of completing tasks.

Deep learning is essentially a process of continuous feature description, that is, a hierarchical feature extraction process and the more features that are extracted and the more accurate, the easier it is to achieve overfitting. The fewer and more fuzzy the feature extraction, the easier it is to achieve underfitting, and connecting the network structure is one of the most basic methods in deep learning networks. Therefore, how to use massive data to train model parameters so that the training results reach the peak accuracy is the difficulty of deep learning. In the early days, it was often used for feature extraction and classification and recognition, but its adjacent layers were closely related and demanded a large amount of storage space and computing space. The main function of generating a confrontation network is to generate confrontation samples [20]. Compared with other models, its advantage is that it can generate better samples, can train any kind of generator network, and can effectively avoid the problems of its process and approximate calculation probability. Its main function is to perform feature extraction and the advantage is that it can model the content of the sequence. The disadvantage is that there are many training parameters and inconvenient adjustment, gradient explosion, and gradient dissipation may occur, and the network does not have the ability to learn features. After the last feature extractor, all the feature maps are expanded and arranged into a one-dimensional feature vector, which is used as the input connection classifier [21].

From the perspective of cognitive psychology, children's painting process is divided into three stages: perception, reflection, and production. The perception stage is the process in which children feel the shape, color, light, space, and other attributes of the painted object and the integrity of the complete image formed by the painting activity. After this, children will psychologically process and reform the aesthetic psychological images acquired through perception. This process of psychological 


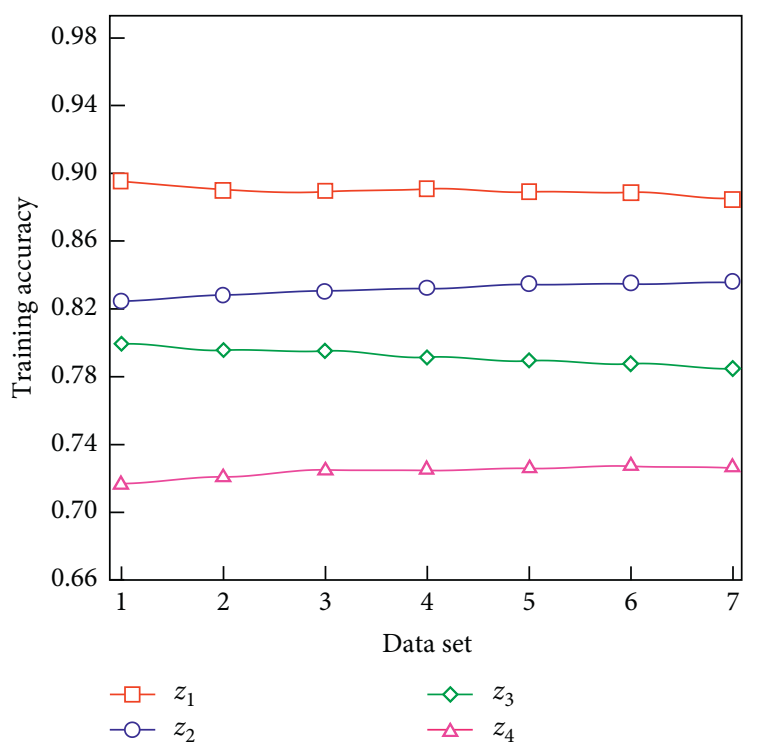

(a)

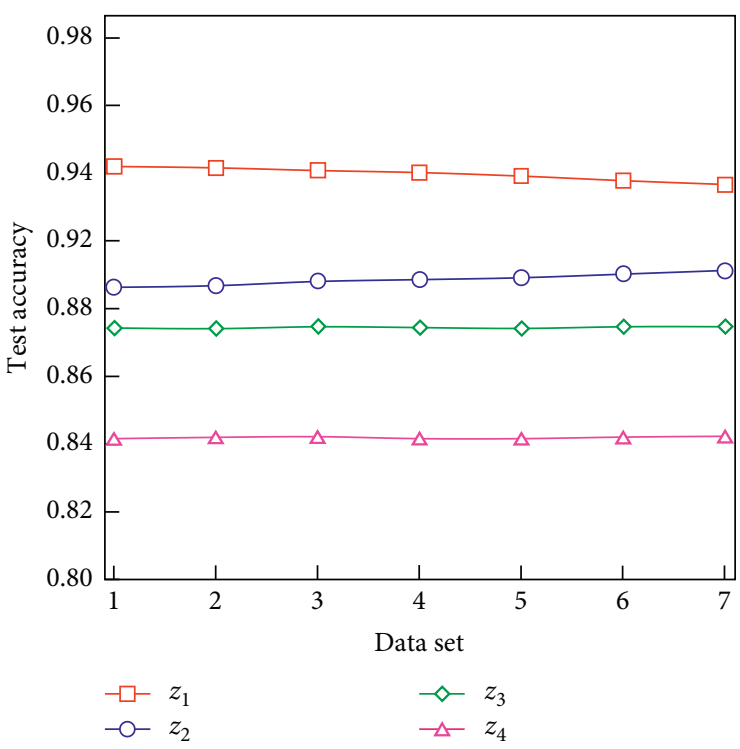

(b)

FIgURE 5: Matching training accuracy and test accuracy of four typical children's psychological distresses. $z_{1}$, solitary scene matching; $z_{2}$, social scene matching; $z_{3}$, entertainment scene matching; $z_{4}$, learning scene matching.

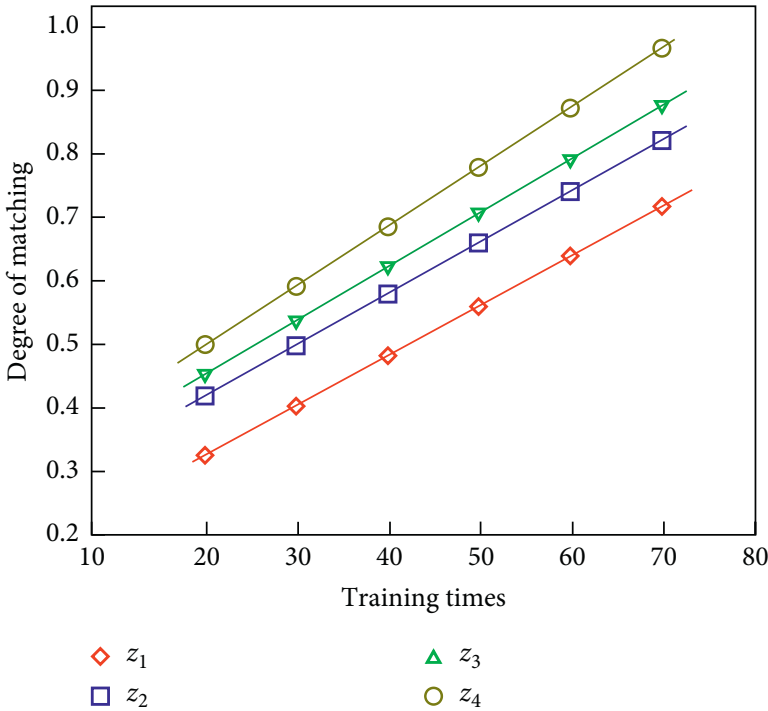

(a)

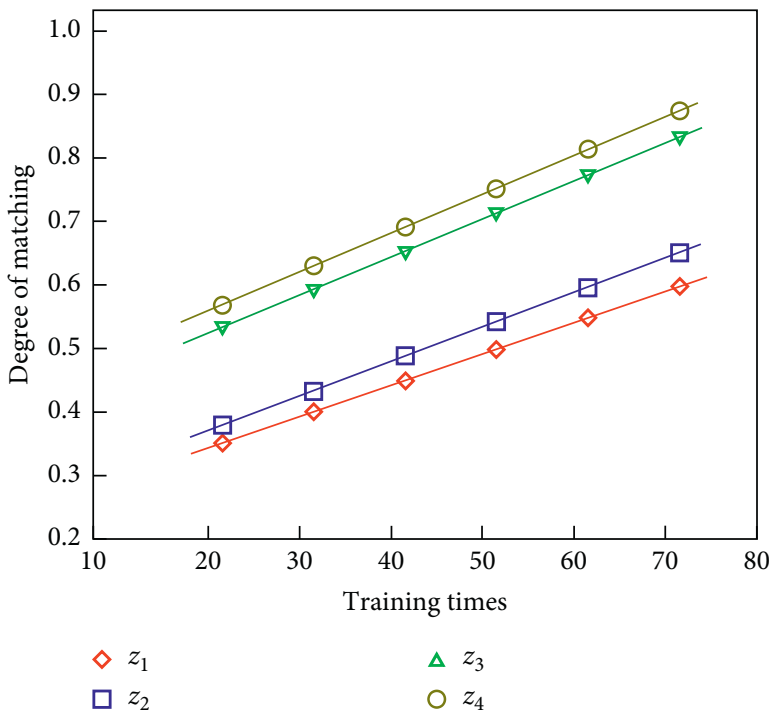

(b)

FIGURE 6: (a) Degree of matching of four typical children's psychological distresses using deep spatiotemporal feature extraction algorithm and (b) dynamic scene understanding algorithm; $z_{1}$, solitary scene matching; $z_{2}$, social scene matching; $z_{3}$, entertainment scene matching; $z_{4}$, learning scene matching.

processing and transformation is also the stage of reflection [22]. The production stage is the result of children's introspection, and the initial painting is formed by using painting tools and materials such as paper and pen, as well as artistic language such as modeling and composition. The above scene matching process needs to be repeated and includes modification and correction of the created content and children's attention is very easy to be attracted or diverted. When the entire interaction process is relatively boring, children's attention can easily be transferred to other places and children's attention can be attracted through sounds, animations, and so on. Children's ability to remember, maintain, re-recognize, and reproduce the content and experience reflected by objective things is very weak, so the transfer between different physical states in the scene matching design should be as simple as possible. 


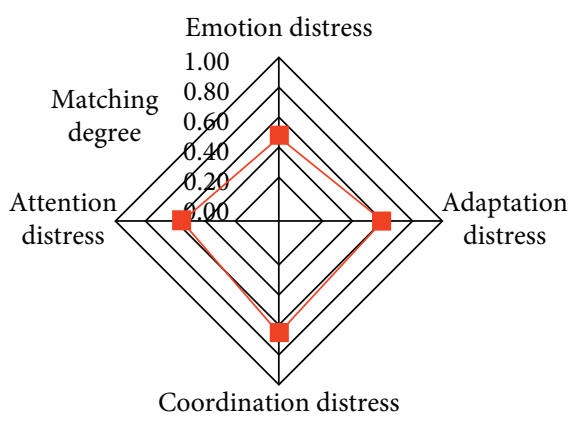

(a)

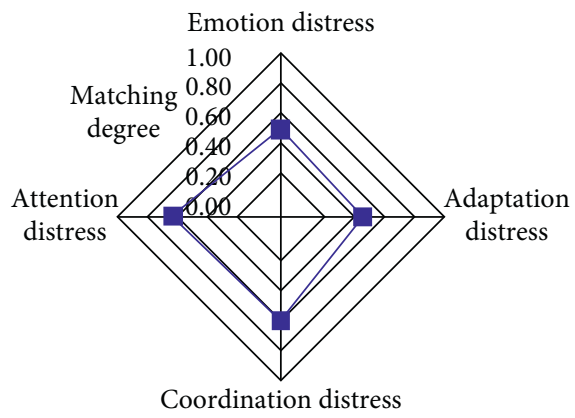

(c)

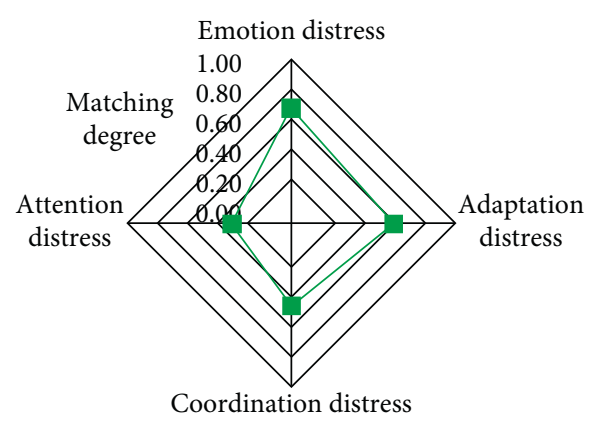

(b)

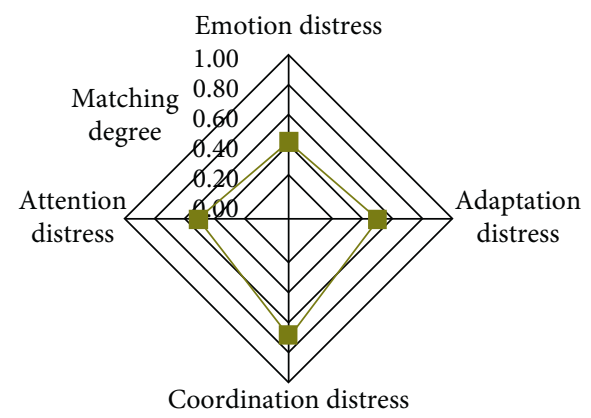

(d)

Figure 7: Matching degrees of children's different psychological distress types in (a) solitary, (b) social, (c) entertainment, and (d) learning scene matching.

\section{Conclusions}

This paper constructed a scene matching model for children's psychological distress based on deep learning algorithm, analyzed the scene feature extraction and matching function construction of children's psychological distress, proposed a scene matching method for children's psychological distress based on deep learning algorithm, performed scene feature matching and information processing of children's psychological distress, and finally conduced a simulation experiment and analyzed its results. Deep learning is essentially a process of continuous feature description, that is, a hierarchical feature extraction process. The more features that are extracted and more accurate, the easier it is to achieve overfitting; the fewer and more fuzzy the feature extraction, the easier it is to achieve underfitting. In scene matching, when children complete a task alone or in a team, they must face physical difficulties during their growth, and this is good for the establishment of their independent personality, and a good scene matching will also help. The degree of confidence network model can be carried out through unsupervised training in the pretraining stage, which does not require a large amount of label data, which greatly improves the adaptability of the model; in the pretraining process, the selection of parameters can speed up the convergence and reduce the computational complexity. The role image of children is constantly being institutionalized into the image of the educated and in the parent-child relationship. The interaction between parents and children is not only educational activities, but also parenting activities and games. This requires designers to design systematic scene matching from the perspective of children in the process of design and at the same time considers more humanistic care and truly considers how the system can better meet the needs of children. The results show that the deep learning algorithm can have a deep and abstract mining on the characteristics of children's psychological distress scenes and obtain a large amount of more representative characteristic information through training on large-scale data, thereby improving the accuracy of classification and matching of children's psychological distress scenes. The study results of this paper provide a reference for further researches on the scene matching method for children's psychological distress based on deep learning algorithm.

\section{Data Availability}

The data used to support the findings of this study are available from the corresponding author upon request.

\section{Conflicts of Interest}

The authors declare that they have no known competing financial interests or personal relationships that could have appeared to influence the work reported in this paper.

\section{References}

[1] R. Hannonen, K. Eklund, A. Tolvanen et al., "Psychological distress of children with early-onset type 1 diabetes and their mothers' well-being," Acta Paediatrica, vol. 104, no. 11, pp. 1144-1149, 2015. 
[2] L. Rojas-Flores, M. L. Clements, J. Hwang Koo, and J. London, "Trauma and psychological distress in Latino citizen children following parental detention and deportation," Psychological Trauma: Theory, Research, Practice, and Policy, vol. 9, no. 3, pp. 352-361, 2017.

[3] R. Riahi, M. E. Motlagh, R. Heshmat, M. Qorbani, S. S. Daniali, and R. Kelishadi, "Body weight misperception and psychological distress among children and adolescents: the CASPIAN-V study," Osong Public Health and Research Perspectives, vol. 10, no. 5, pp. 315-324, 2019.

[4] I. Yorke, P. White, A. Weston, M. Rafla, T. Charman, and E. Simonoff, "The association between emotional and behavioral problems in children with autism spectrum disorder and psychological distress in their parents: a systematic review and meta-analysis," Journal of Autism and Developmental Disorders, vol. 48, no. 10, pp. 3393-3415, 2018.

[5] B. Shi, X. Bai, and C. Yao, "An end-to-end trainable neural network for image-based sequence recognition and its application to scene text recognition," IEEE Transactions on Pattern Analysis and Machine Intelligence, vol. 39, no. 11, pp. 2298-2304, 2017.

[6] G. Cheng, C. Yang, X. Yao, L. Guo, and J. Han, "When deep learning meets metric learning: remote sensing image scene classification via learning discriminative CNNs," IEEE Transactions on Geoscience and Remote Sensing, vol. 56, no. 5, pp. 2811-2821, 2018.

[7] J. Li, X. Mei, D. Prokhorov, and D. Tao, "Deep neural network for structural prediction and lane detection in traffic scene," IEEE Transactions on Neural Networks and Learning Systems, vol. 28, no. 3, pp. 690-703, 2017.

[8] K. Hammernik, T. Klatzer, E. Kobler et al., "Learning a variational network for reconstruction of accelerated MRI data," Magnetic Resonance in Medicine, vol. 79, no. 6, pp. 3055-3071, 2018.

[9] O. A. Umoren and N. C. Owiriwa, "Influence of domestic violence and parenting style on children's psychological wellbeing," Gender and Behaviour, vol. 16, no. 3, pp. 12246-12254, 2018.

[10] Y.-P. Hsieh, H.-S. Wei, H.-L. Hwa, A. C.-T. Shen, J.-Y. Feng, and C.-Y. Huang, "The effects of peer victimization on children's Internet addiction and psychological distress: the moderating roles of emotional and social intelligence," Journal of Child and Family Studies, vol. 28, no. 9, pp. 2487-2498, 2019.

[11] G. Y. K. Ma and W. W. S. Mak, "Caregiving-specific worry, affiliate stigma, and perceived social support on psychological distress of caregivers of children with physical disability in Hong Kong," American Journal of Orthopsychiatry, vol. 86, no. 4, pp. 436-446, 2016.

[12] C. Due, N. Heer, M. Baak, and S. Hanson-Easey, “"At night he cries from dreams": perceptions of children's psychological distress and wellbeing amongst parents with refugee or asylum seeker backgrounds in Australia," Australian Psychologist, vol. 54, no. 5, pp. 438-449, 2019.

[13] Q. Zou, L. Ni, T. Zhang, and Q. Wang, "Deep learning based feature selection for remote sensing scene classification," IEEE Geoscience and Remote Sensing Letters, vol. 12, no. 11, pp. 2321-2325, 2015.

[14] M. Mizumoto, "Examination of the relationships between parents and their emerging adult children," The Japanese Journal of Educational Psychology, vol. 66, no. 2, pp. 111-126, 2018.

[15] S. Ji, W. Xu, M. Yang, and K. Yu, "3D convolutional neural networks for human action recognition," IEEE Transactions on Pattern Analysis and Machine Intelligence, vol. 35, no. 1, pp. 221-231, 2013.

[16] S. S. Han, M. S. Kim, W. Lim, G. H. Park, I. Park, and S. E. Chang, "Classification of the clinical images for benign and malignant cutaneous tumors using a deep learning algorithm," Journal of Investigative Dermatology, vol. 138, no. 7, pp. 1529-1538, 2018.

[17] S. Shokri, Z. Khanjani, T. Hashemi, and K. Esmaeilpuor, "Comparison of parenting stress and psychological distress in mothers of children with autism disorder and developmental delay," Quarterly Journal of Child Mental Health, vol. 4, no. 3, pp. 79-90, 2017.

[18] S. Ren, K. He, R. Girshick, and J. Sun, "Faster R-CNN: towards real-time object detection with region proposal networks," IEEE Transactions on Pattern Analysis and Machine Intelligence, vol. 39, no. 6, pp. 1137-1149, 2017.

[19] P. N. Doku and H. Minnis, "Multi-informant perspective on psychological distress among Ghanaian orphans and vulnerable children within the context of HIV/AIDS," Psychological Medicine, vol. 46, no. 11, pp. 2329-2336, 2016.

[20] V. Khamis, "Coping with war trauma and psychological distress among school-age Palestinian children," American Journal of Orthopsychiatry, vol. 85, no. 1, pp. 72-79, 2015.

[21] J. Jones, A. S. Nowacki, A. Greene, C. Traul, and J. Goldfarb, "Investigating parent needs, participation, and psychological distress in the children's hospital," Hospital Pediatrics, vol. 7, no. 7, pp. 385-394, 2017.

[22] H. H. Musa and S. Yohanna, "Effect of family counsellling on psychological distress among caregivers of children with sickle cell anaemia in a Tertiary Hospital in North Central Nigeria," Nigerian Journal of Family Practice, vol. 8, no. 2, pp. 49-56, 2017. 